\title{
STRAIGHTFORWARD DISCRETISATION OF GREEN FUNCTION AND FREE-SURFACE POTENTIAL FLOW AROUND A THREE-DIMENSIONAL LIFTING BODY
}

\author{
ZHI-MIN CHEN, QIANG LIU, AND W. G. PRICE
}

\begin{abstract}
A straightforward evaluation approach of free-surface Green function is developed to solve the potential flow around a three-dimensional lifting body. The free-surface waves generated by the movement of the lifting body is presented in an expansion of plane regular waves traveling in $\theta$ directions with wave number magnitudes $k>0$. A boundary element method is combined with the evaluation approach and Hess-Smith panel integral formulae to predict hydrodynamic performance of a three-dimensional lifting body. $\mathrm{Nu}$ merical results produced by the proposed method are compared favourably with experimental measurements.
\end{abstract}

Keywords: Finite boundary element method, harmonic function, lifting body, three-dimensional free-surface Green function

\section{INTRODUCTION}

In ship hydrodynamics, the understanding of free-surface phenomena is a fundamental issue. To this end, in a potential flow analysis, Havelock [2] introduced a free-surface Green function or a free-surface source potential to describe the free-surface wave generated from the fluid-structure interaction between a body and uniform stream. In deriving the solution of the velocity potential describing this problem, the associated linear free-surface boundary equation is automatically solved in the free-surface Green function scheme by eliminating the infinite freesurface boundary integral from the fluid boundary integral formulation.

The free-surface Green function consists of the Rankine source potential, its image potential and a singular wave integral $K$ expressed as

$$
G=-\frac{1}{|(x, y, z)-(\xi, \eta, \zeta)|}+\frac{1}{|(x, y,-z)-(\xi, \eta, \zeta)|}-K
$$

For $x-\xi \geq 0$ and $\nu$ the wave number (see the attached Nomenclature for the definition of variables), the singular wave integral $K$ is a harmonic function in the following form $[3,4,5,6]$ :

$$
\begin{aligned}
K= & 4 \nu \int_{0}^{\frac{\pi}{2}} \mathrm{e}^{\nu(z+\zeta) \sec ^{2} \theta} \sin [\nu(x-\xi) \sec \theta] \cos \left[\nu(y-\eta) \sin \theta \sec ^{2} \theta\right] \sec ^{2} \theta d \theta \\
& +\frac{4 \nu}{\pi} \int_{0}^{\frac{\pi}{2}} d \theta \mathrm{PV} \int_{0}^{\infty} \frac{\mathrm{e}^{k(z+\zeta)} \cos [k(x-\xi) \cos \theta]}{k \cos ^{2} \theta-\nu} \cos [k(y-\eta) \sin \theta] d k,
\end{aligned}
$$

which is a combination of a double integral determining near-field free-surface waves and a single wave integral accounting for far-field free-surface waves. In the developed Rankine source panel method discretisation schemes, the Rankine source 
singularity occurring in a panel integral was successfully evaluated $[7,8]$, whereas, the singularity arising in the double integral of $K$ leads to numerical computational difficulties which have been widely discussed $[5,9,10]$.

Havelock $[2,11]$ introduced the regular wave integral

$$
K^{\mu}=\operatorname{Re} \int_{-\frac{\pi}{2}}^{\frac{\pi}{2}} d \theta \int_{0}^{\infty} \frac{2 \nu \mathrm{e}^{k[z+\zeta+\mathrm{i}(x-\xi) \cos \theta+\mathrm{i}(y-\eta) \sin \theta]}}{\pi\left(k \cos ^{2} \theta-\nu-\mathrm{i} \mu \cos \theta\right)} d k .
$$

Using this wave integral $K^{\mu}$, we have the approximation for the singular wave integral $K$ in the form [6]

$$
K=\lim _{\mu \rightarrow 0+} K^{\mu}
$$

For the uniqueness of the wave integral, one may refer to $[12,13]$ for an initial value problem for velocity potential $\mathrm{e}^{\mu t} \phi(x)$.

Recently, two-dimensional free-surface Green function involving a regular wave integral was derived by the author [14]. The regular wave integral replaces the corresponding singular wave integral in numerical approximations ensures that the Green function evaluation can be made straightforwardly by ignoring the wave integral singularity problem and evaluation scheme was applicable to the numerical simulation of two-dimensional potential flows around a lifting body $[14,15]$. The straightforward evaluation using the three-dimensional regular wave integral $K_{\mu}$ was employed to produce numerical solution describing the potential flow around a thin body [16] based on the Michell thin ship theory. This method is also further applied to a numerical solution describing a Hess-Smith panel method based numerical discretisation of the potential flow around a non-lifting body [17]. However, it is unknown if the approach is applicable to fluid motion around a lifting body. To give a confirmative answer to this hydrodynamics problem, we provide further development of the studies $[14,15]$ to predict the pressure distribution and generated free-surface waves experienced by a three-dimensional lifting body, such as a hydrofoil, submerged in a uniform stream. It is shown that a free-surface wave is expressed as an expansion of plane regular waves traveling in the directions $-\frac{\pi}{2}<\theta \leq \frac{\pi}{2}$ with the wave number magnitudes $k>0$. This numerical wave, covering both near field and far field wave parts, is different to the far field free-surface wave approximation by integrating elementary waves in the directions $-\frac{\pi}{2}<\theta \leq \frac{\pi}{2}$ $[18,19]$ with the fixed wave number value $k=\nu$ and the far field free-surface wave approximation by using the single wave integral of (2) (see, for example, [20, 21]). The predictions from the proposed method are shown to be in favourable agreement with experimental measurements $[22,23]$. For other numerical simulation methods on the lifting body problem, one may refer to [24, 25, 26, 27].

For the ship wave problem based on the free-surface Green function, a series of investigations on numerical implementation of the Neumann-Michell theory have been obtained by Noblesse and his co-workers. They derived a new formulation of the line integral around the ship waterline in the boundary integral representation of the velocity potential [28], provided numerical smoothing of the flow velocity and numerical filtering of short waves to present better wave predictions [29], and showed the efficiency of a simple ship wave approximation formulation of the Neumann-Michell theory [30].

In the present study, free-surface Green function panel method is employed so that the free-surface boundary condition is automatically satisfied. With the use 
of (4), the singular wave integral $K$ is replaced by $K^{\mu}$ for small $\mu>0$ in numerical simulations. However, the methods using the Rankine source potential rather than the free-surface Green function due to the singularity problem of $K$ have been well developed. Rankine source panel methods focus mainly on solving free-surface boundary equations in which the free source potential is solved simultaneously with the body surface potential and radiation condition. For example, [10] utilized a finite-difference method to solve free-surface boundary equation; $[31,32]$ used B-spline functions to approximate the near field free-surface boundary equation solution and periodic free-surface eigenfunctions to satisfy the far field radiation condition; [33, 34] combined a B-spline function approximation, Fourier integral and dispersion relations to solve free-surface boundary equations. Although Rankine source panel methods require much larger computing memory to solve free-surface boundary conditions, they provide more flexibility to tackle nonlinear fluid boundary equations [35] through a developed mixed Euler-Lagrange iteration scheme [36, 37].

\section{Nomenclature}

$b:-$ hydrofoil span

$c:$ - hydrofoil chord

$C_{d}:-$ drag coefficient

$C_{l}:-$ lift coefficient

$C_{p}:-$ pressure coefficient

$\left(x^{\prime}, y^{\prime}, z^{\prime}\right)$ : - dimensional coordinates

$(x, y, z):$-non-dimensional coordinates

$c_{l, m}$ : - regular wave expansion coefficients

Fn: - Froude number $U / \sqrt{g c}$

$h$ : (NACA4412) - vertical distance between $z=0$ and a mid chord point

$h$ : (Joukowski) - vertical distance between $z=0$ and a trailing edge point

$g$ : - gravitational acceleration

$G$ : - free-surface Green function

$G^{\mu}$ : - dissipative free-surface Green function

i: - pure imaginary number $\sqrt{-1}$

$K$ : - singular wave integral

$K^{\mu}$ : - regular wave integral

$\mathbf{n}_{i, j}:$ - panel normal vector

$N_{c}$ : - number of panels in chordwise direction

$N_{s}$ : - number of panels in the spanwise direction

$N_{k}:-$ number of panels of $\left(0, t_{\max }\right)$

$\left(0, t_{\max }\right):-$ approximate domain of the wave number integral domain $(0, \infty)$

$N_{\theta}$ : - number of panels of the $\theta$ direction domain $\left(-\frac{\pi}{2}, \frac{\pi}{2}\right)$

$\mathbf{p}_{i, j}:-$ panel grid points

$\mathbf{q}_{i, j}:$ - panel control points

$U:-$ uniform stream speed

$\mathbf{t}_{i, j}^{c}:$ - panel chordwsie tangential vector

$\mathbf{t}_{i, j}^{s}:$ - panel spanwsie tangential vector

$D:$ - linearised fluid domain

$\alpha$ : -angle of attack

$\mu$ : - energy dissipation number 
$\nu$ : -wave number $1 / \mathrm{Fn}^{2}$

$\phi:-$ dimensionless perturbed velocity potential

$\chi$ : -wave elevation around a hydrofoil

$\chi_{s}$ : -wave elevation around a single source

\section{Regular WAVE INTEGRAL}

Let $\left(x^{\prime}, y^{\prime}, z^{\prime}\right)$ be the dimensional coordinates of a coordinate system with origin, $O$, in the calm water surface defined by the plane $z^{\prime}=0$, which is the upper bound of the linear fluid domain as shown in Figure 3.1. Let $\left(\xi^{\prime}, \eta^{\prime}, \zeta^{\prime}\right)$ denote a point source in a uniform stream of speed $U$ moving in the positive $O x$ direction. The corresponding non-dimensional coordinates are defined as $(x, y, z)=\left(x^{\prime}, y^{\prime}, z^{\prime}\right) / c$ where $c$ denotes the hydrofoil chord length. Similarly, $(\xi, \eta, \zeta)=\left(\xi{ }^{\prime}, \eta^{\prime}, \zeta^{\prime}\right) / c$ defines a non-dimensional source point. Thus the source potential of $(\xi, \eta, \zeta)$ is the free-surface Green function defined by (1), (3) and (4). Instead of approximating the singular wave integral $K$ in (2) after taking the limit (4) in earlier investigations such as [6], we use the approximation

$$
K \approx K^{\mu} \text { for small } \mu
$$

before taking the limit (4) and expanding $K^{\mu}$ into a sum of whole space harmonic functions [16].

Since the singularity of $K^{\mu}$ is removed because $\mu>0$, we may evaluate the regular wave integral $K^{\mu}$ over a set of mesh grid points $\left(k_{l}, \theta_{m}\right)\left(1 \leq l \leq N_{k}+1\right.$, $\left.1 \leq m \leq N_{\theta}+1\right)$ covering the integration domain $(0, \infty) \times\left(-\frac{\pi}{2}, \frac{\pi}{2}\right)$ for suitable integers $N_{k}$ and $N_{\theta}$. As adopted in [16], letting the exponential function

$$
\mathrm{e}^{k[z+\zeta+\mathrm{i}(x-\xi) \cos \theta+\mathrm{i}(y-\eta) \sin \theta]}
$$

be constant with respect to $k$ and $\theta$ in every cell, the discretisation of the regular wave integral $K^{\mu}$ takes the form

$$
\begin{aligned}
& \frac{2 \nu}{\pi} \operatorname{Re} \sum_{l=1 m=1}^{N_{k}} \sum_{k_{l}}^{N_{\theta}} \int_{\theta_{m}}^{k_{l+1}} d k \int_{\theta_{m+1}}^{\theta^{k[z+\zeta+\mathrm{i}(x-\xi) \cos \theta+\mathrm{i}(y-\eta) \sin \theta]}} \frac{2 \cos ^{2} \theta-\nu-\mathrm{i} \mu \cos \theta}{\pi} d \theta \\
& \quad=\frac{2 \nu}{\operatorname{Re}} \sum_{l=1}^{N_{k}} \sum_{m=1}^{N_{\theta}} c_{l, m} \mathrm{e}^{k_{l}\left[z+\zeta+\mathrm{i}(x-\xi) \cos \theta_{m}+\mathrm{i}(y-\eta) \sin \theta_{m}\right]}
\end{aligned}
$$

This is an expansion covering whole space harmonic functions with the expansion coefficients defined by

$$
c_{l, m}=\ln \frac{k_{l+1} \cos ^{2} \theta_{m}-\nu-\mathrm{i} \mu \cos \theta_{m}}{k_{l} \cos ^{2} \theta_{m}-\nu-\mathrm{i} \mu \cos \theta_{m}}\left[\tan \theta_{m+1}-\tan \theta_{m}\right] .
$$

Here the continuous function

$$
\frac{\cos ^{2} \theta \mathrm{e}^{k[z+\zeta+\mathrm{i}(x-\xi) \cos \theta+\mathrm{i}(y-\eta) \sin \theta]}}{k \cos ^{2} \theta-\nu-\mathrm{i} \mu \cos \theta}
$$

with respect to $\theta$ on the interval $\left[\theta_{m}, \theta_{m+1}\right]$ is evaluated at $\theta=\theta_{m}$. The evaluation can also be made for $\theta=\frac{1}{2}\left(\theta_{m}+\theta_{m+1}\right)$ and $\theta=\theta_{m+1}$, respectively. This expansion gives rise to a similar expansion of the derivative of $K^{\mu}$ in the direction of a vector 


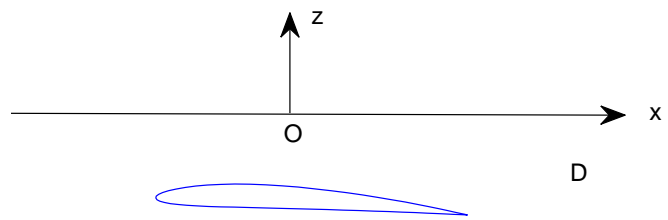

FiguRE 1. The coordinate frame of reference with origin, $O$, projected on the central plane $y=0$.

$\left(v_{1}, v_{2}, v_{3}\right)$ in the following form

$$
\begin{aligned}
& v_{1} \frac{\partial K^{\mu}}{\partial \xi}+v_{2} \frac{\partial K^{\mu}}{\partial \eta}+v_{3} \frac{\partial K^{\mu}}{\partial \zeta} \\
& =\frac{2 \nu}{\pi} \operatorname{Re} \sum_{l=1}^{N_{k}} \sum_{m=1}^{N_{\theta}} c_{l, m} k_{l}\left(v_{3}-\mathrm{i} v_{1} \cos \theta_{m}-\mathrm{i} v_{2} \sin \theta_{m}\right) \\
& \cdot \mathrm{e}^{k_{l}\left[z+\zeta+\mathrm{i}(x-\xi) \cos \theta_{m}+\mathrm{i}(y-\eta) \sin \theta_{m}\right]} .
\end{aligned}
$$

\section{PANEL Method}

As an example to demonstrate the application of the expansion technique to a lifting body problem, the numerical simulation by using a panel method is demonstrated for the potential flow around a three-dimensional hydrofoil in a uniform stream.

3.1. Boundary integral formulation. A three-dimensional hydrofoil of chord length $c$ is submerged in a uniform stream of speed $U$. The water depth of the fluid is infinite. $D$ denotes the linearized fluid domain upper bounded by the calm water surface $z=0$. The non-dimensional coordinate frame of the vertical plane $y=0$ is illustrated in Figure 3.1. The total dimensional velocity potential of the hydrofoil problem is described as

$$
\hat{\Phi}=U c x+U c \phi .
$$

It is a harmonic function in the fluid domain $D$ satisfying the solid boundary condition

$$
\mathbf{n} \cdot \nabla \hat{\Phi}=U c \mathbf{n} \cdot \nabla(x+\phi)=0 \text { on } S,
$$

where $S$ is the hydrofoil surface, $\mathbf{n}$ is the unit normal vector field of $S$ pointing into the fluid domain and the dimensionless function $\phi$ denotes the perturbed velocity potential subject to the linear dissipative free-surface boundary condition

$$
\frac{\partial^{2} \phi}{\partial x^{2}}+\nu \frac{\partial \phi}{\partial z}+\mu \frac{\partial \phi}{\partial x}=0 \quad \text { on } \quad z=0
$$

Using the three-dimensional dissipative free-surface Green function

$$
G^{\mu}=\frac{-1}{|(x, y, z)-(\xi, \eta, \zeta)|}+\frac{1}{|(x, y,-z)-(\xi, \eta, \zeta)|}-K^{\mu}
$$

and boundary condition (10), we find that the velocity potential $\phi$ can be presented as a solution of the boundary integral equation over the hydrofoil surface $S$ and the 
upper and lower surfaces $S_{w}^{ \pm}$of an infinite wake cut. That is,

$$
\begin{aligned}
& 4 \pi \phi(\mathbf{q})+\int_{S \cup S_{w}^{+} \cup S_{w}^{-}} \phi(\mathbf{p}) \mathbf{n} \cdot \nabla G^{\mu}(\mathbf{q}, \mathbf{p}) d s_{\mathbf{p}} \\
& \quad=\int_{S} G^{\mu}(\mathbf{q}, \mathbf{p}) \mathbf{n} \cdot \nabla \phi(\mathbf{p}) d s_{\mathbf{p}}
\end{aligned}
$$

for $\mathbf{q}=(x, y, z) \in D, \mathbf{p}=(\xi, \eta, \zeta) \in S \cup S_{w}^{+} \cup S_{w}^{-}, \nabla=\left(\partial_{\xi}, \partial_{\eta}, \partial_{\zeta}\right)$ and $\mathbf{n}=\mathbf{n}(\mathbf{p})$ is the normal vector field of $S \cup S_{w}^{+} \cup S_{w}^{-}$pointing into the fluid domain.

The equation (12) is only involved in the description of the body boundary integral and the linear free-surface boundary condition, implied in the Green function, is not required to solve (12). The velocity potential solution is solved by using the solid boundary condition (9) and a Kutta condition for controlling the integral over the wake cut. The derivation of the Green function or source potential is crucially dependent on the source being totally submerged below the calm water surface at depth $h$. It is found that increasing this depth, $h$, leads to an increase in computational accuracy.

3.2. Discretisation of the boundary integral equation (12). For simplicity, to demonstrate the discretisation process, a three-dimensional hydrofoil of uniform chord is selected with grid points $\mathbf{p}_{i, j}$ for $i=1, \ldots, N_{c}+1$ and $j=1, \ldots, N_{s}+1$. Here $N_{c}$ denotes the number of panels in the chordwise direction and $N_{c}$ denotes the number of panels in the spanwise direction. The grid points $\mathbf{p}_{1, j}=\mathbf{p}_{N_{c}+1, j}$ represent the trailing edge. The ending grid points of the infinite wake cut panels are

$$
\mathbf{p}_{N_{c}+2, j}=\mathbf{p}_{N_{c}+1, j}+(\lambda, 0,-\lambda)
$$

for $\lambda \rightarrow+\infty$ and $j=1, \ldots, N_{s}+1$.

Therefore, a flat panel with centroid $\mathbf{q}_{i, j}$ is denoted as panel ${ }_{i, j}$ and is defined by the four vertices

$$
\mathbf{p}_{i, j}, \mathbf{p}_{i+1, j}, \mathbf{p}_{i, j+1}, \mathbf{p}_{i+1, j+1},
$$

The local frame referencing the panel $_{i, j}$ consists of the tangential vectors

$$
\begin{aligned}
& \mathbf{t}_{i, j}^{c}= \begin{cases}\frac{\mathbf{p}_{i+1, j}-\mathbf{p}_{i, j}}{\left|\mathbf{p}_{i+1, j}-\mathbf{p}_{i, j}\right|} & \text { for } i=1, \ldots, N_{c}, j=1, \ldots, N_{s}, \\
\frac{1}{\sqrt{2}}(1,0,-1) & \text { for } i=N_{c}+1, j=1, \ldots, N_{s}\end{cases} \\
& \mathbf{t}_{i, j}^{s}=\frac{\mathbf{p}_{i, j+1}-\mathbf{p}_{i, j}}{\left|\mathbf{p}_{i, j+1}-\mathbf{p}_{i, j}\right|}=(0,1,0)
\end{aligned}
$$

for $i=1, \ldots, N_{c}+1, j=1, \ldots, N_{s}+1$, and the normal vector

$$
\mathbf{n}_{i, j}=\mathbf{t}_{i, j}^{c} \times \mathbf{t}_{i, j}^{s} \text { for } i=1, \ldots, N_{c}+1, j=1, \ldots, N_{s} .
$$

Let us assume that $\phi$ is constant on each panel and is defined as $\phi_{i, j}$. On the wake cut, we set the values

$$
\phi_{N_{c}+1, j}=\phi_{N_{c}+1, j}^{+}-\phi_{N_{c}+1, j}^{-} \text {on } \operatorname{panel}_{N_{c}+1, j},
$$

where $\phi_{N_{c}+1, j}^{+}$and $\phi_{N_{c}+1, j}^{-}$represent respectively the constant values of $\phi$ on the upper and lower surfaces of panel $_{N_{c}+1, j}$. The boundary integral equation (12) can 
be discretised for $I=1, \ldots, N_{c}$ and $J=1, \ldots, N_{s}$, into the form

$$
\begin{aligned}
& \phi_{I, J}+\sum_{i=1}^{N_{c}+1 N_{s}} \sum_{j=1} \phi_{i, j} \lim _{\mathbf{q} \in D, \mathbf{q} \rightarrow \mathbf{q}_{I, J}} \int_{\operatorname{panel}_{i, j}} \frac{\mathbf{n}_{i, j} \cdot \nabla G^{\mu}(\mathbf{q}, \mathbf{p})}{4 \pi} d s_{\mathbf{p}} \\
& =-\sum_{i=1}^{N_{c}} \sum_{j=1}^{N_{s}} \mathbf{n}_{i, j} \cdot(1,0,0) \int_{\operatorname{panel}_{i, j}} \frac{G^{\mu}\left(\mathbf{q}_{I, J}, \mathbf{p}\right)}{4 \pi} d s_{\mathbf{p}},
\end{aligned}
$$

after the application of the impermeable solid boundary condition (9) or

$$
\mathbf{n}_{i, j} \cdot \nabla(x+\phi)=0 \text { on } \text { panel }_{i, j} .
$$

To solve the unknowns $\phi_{i, j}$ from the discretisation equation (13), it is necessary to evaluate the panel integrals

$$
\lim _{\mathbf{q} \in D, \mathbf{q} \rightarrow \mathbf{q}_{I, J}} \frac{1}{4 \pi} \int_{\text {panel }_{i, j}} \mathbf{n}_{i, j} \cdot \nabla G^{\mu}(\mathbf{q}, \mathbf{p}) d s_{\mathbf{p}}
$$

and

$$
\frac{1}{4 \pi} \int_{\text {panel }_{i, j}} G^{\mu}\left(\mathbf{q}_{I, J}, \mathbf{p}\right) d s_{\mathbf{p}}
$$

with respect to $G^{\mu}$ in (11).

The panel integrals for the Rankine source potential, its image and their normal derivatives are evaluated by the integration formulae $[7,8]$. These were also presented by [38] resulting from the Gauss-Bonnet theorem. The integrations, $[7,8]$, are over a flat panel in $(\xi, \eta)$ plane and the control point of the panel is the origan $(0,0)$. Therefore, for the integrals over the panel $_{i, j}$ with respect to Rankine source and its image, it is necessary to transfer the points $\mathbf{p}, \mathbf{p}_{i, j}, \mathbf{q}_{i, j}$ and $\mathbf{q}_{I, J}$ in the global frame into the local frame $\mathbf{t}_{i, j}^{c}, \mathbf{t}_{i, j}^{s}$ and $\mathbf{n}_{i, j}$.

With the use of the expansions (5)-(7) and adopting the notation $\mathbf{q}_{I, J}=(x, y, z)$ and

$$
\mathbf{p}_{i, j}=\left(\xi_{i, j}, \eta_{i, j}, \zeta_{i, j}\right), \mathbf{t}_{i, j}^{c}=\left(t_{i, j, 1}, t_{i, j, 2}, t_{i, j, 3}\right)
$$

and

$$
\mathbf{n}_{i, j}=\left(n_{i, j, 1}, n_{i, j, 2}, n_{i, j, 3}\right)
$$

the panel integrals associated with the regular wave integral $K^{\mu}$ are calculated from the expressions

$$
\begin{aligned}
\int_{\text {panel }_{i, j}} & \frac{1}{4 \pi} K^{\mu}\left(\mathbf{q}_{I, J}, \mathbf{p}\right) d s_{\mathbf{p}} \\
= & \frac{\nu}{2 \pi^{2}} \operatorname{Re} \sum_{l=1}^{N_{k}} \sum_{m=1}^{N_{\theta}} c_{l, m} \int_{\mathbf{p}_{i, j}}^{\mathbf{p}_{i+1, j}} \int_{\mathbf{p}_{i, j}}^{\mathbf{p}_{i, j+1}} \\
= & \mathrm{e}^{k_{l+1}\left[z+\zeta+\mathrm{i}(x-\xi) \cos \theta_{m}+\mathrm{i}(y-\eta) \sin \theta_{m}\right]} d s \\
& \sum_{l=1 m=1}^{N_{k}} \sum^{N_{\theta}} \frac{\nu c_{l, m}}{2 \pi^{2}} \frac{\mathrm{e}^{\mathrm{i} k_{l+1}\left(y-\eta_{i, j+1}\right) \sin \theta_{m}}-\mathrm{e}^{\mathrm{i} k_{l+1}\left(y-\eta_{i, j}\right) \sin \theta_{m}}}{-\mathrm{i} k_{l+1} \sin \theta_{m}} \\
& \frac{\mathrm{e}^{k_{l+1}\left[z+\zeta_{i+1, j}+\mathrm{i}\left(x-\xi_{i+1, j}\right) \cos \theta_{m}\right]}-\mathrm{e}^{k_{l+1}\left[z+\zeta_{i, j}+\mathrm{i}\left(x-\xi_{i, j}\right) \cos \theta_{m}\right]}}{k_{l+1}\left(t_{i, j, 3}-\mathrm{i} t_{i, j, 1} \cos \theta_{m}\right)}
\end{aligned}
$$


and

$$
\begin{aligned}
& \frac{1}{4 \pi} \int_{\text {panel }_{i j}} \mathbf{n}_{i, j} \cdot \nabla K^{\mu}\left(\mathbf{q}_{I, J}, \mathbf{p}\right) d s \\
& =\operatorname{Re} \sum_{l=1}^{N_{k}} \sum_{m=1}^{N_{\theta}} \frac{\nu c_{l, m}}{2 \pi^{2}} \int_{\mathbf{p}_{i, j}}^{\mathbf{p}_{i+1, j}} \int_{\mathbf{p}_{i, j}}^{\mathbf{p}_{i, j+1}} k_{l+1}\left[n_{i, j, 3}-\mathrm{i} n_{i, j, 1} \cos \theta_{m}\right] \\
& \cdot \mathrm{e}^{k_{l+1}\left[z+\zeta+\mathrm{i}(x-\xi) \cos \theta_{m}+\mathrm{i}(y-\eta) \sin \theta_{m}\right]} d s \\
& \left.=\frac{\nu}{2 \pi^{2}} \operatorname{Re} \sum_{l=1 m=1}^{N_{k}} \sum_{l, m}^{N_{\theta}} c_{i, j, 3}-\mathrm{i} n_{i, j, 1} \cos \theta_{m}\right] \\
& \cdot \frac{\mathrm{e}^{k_{l+1}\left[z+\zeta_{i+1, j}+\mathrm{i}\left(x-\xi_{i+1, j}\right) \cos \theta_{m}\right]}-\mathrm{e}^{k_{l+1}\left[z+\zeta_{i, j}+\mathrm{i}\left(x-\xi_{i, j}\right) \cos \theta_{m}\right]}}{t_{i, j, 3}-\mathrm{i} t_{i, j, 1} \cos \theta_{m}} \\
& \frac{\mathrm{e}^{\mathrm{i} k_{l+1}\left(y-\eta_{i, j+1}\right) \sin \theta_{m}}-\mathrm{e}^{\mathrm{i} k_{l+1}\left(y-\eta_{i, j}\right) \sin \theta_{m}}}{-\mathrm{i} k_{l+1} \sin \theta_{m}}
\end{aligned}
$$

\section{Numerical Simulation FOR PRESSURE FORCES}

In the previous section, the panel integrals occurring in (15)-(16) follow from the Hess-Smith panel integral formulae [7] and the results arising from the regular wave integral evaluation (18)-(19). It therefore follows that the boundary integral discretisation equations (13) can be rewritten as

$$
\phi_{I, J}+\sum_{i=1}^{N_{c}+1} \sum_{j=1}^{N_{s}} \phi_{i, j} a_{I, J, i, j}=B_{I, J}
$$

for $I=1, \ldots, N_{c}, J=1, \ldots, N_{s}$, where $a_{I, J, i, j}$ denotes the integral (15) and $B_{I, J}$ denotes the right-hand side of (13).

The algebraic equations system (20) is not complete as the extra unknowns $\phi_{N_{c}+1, j}$ are to be specified. To do so, we use the Kutta condition [41] that the chordwise tangential velocity at the trailing edge on the upper surface is opposite to that on the lower surface:

$$
\phi_{N_{c}+1, j}^{+}-\phi_{N_{c}, j}=-\left(\phi_{1, j}-\phi_{N_{c}+1, j}^{-}\right) \text {for } j=1, \ldots, N_{s}
$$

or

$$
\phi_{N_{c}+1, j}=\phi_{N_{c}, j}-\phi_{1, j} \text { for } j=1, \ldots, N_{s} .
$$

Therefore, the substitution of (21) into (20) gives the complete algebraic equations system

$$
\phi_{I, J}+\sum_{i=1}^{N_{c}} \sum_{j=1}^{N_{s}} \phi_{i, j} A_{I, J, i, j}=B_{I, J}
$$

for $I=1, \ldots, N_{c}, \quad J=1, \ldots, N_{s}$ with

$$
A_{I, J, i, j}= \begin{cases}a_{I, J, i, j} & \text { if } 1<i<N_{c}+1, \\ a_{I, J, 1, j}-a_{I, J, N_{c}+1, j} & \text { if } i=1 \\ a_{I, J, N_{c}, j}+a_{I, J, N_{c}+1, j} & \text { if } i=N_{c} .\end{cases}
$$



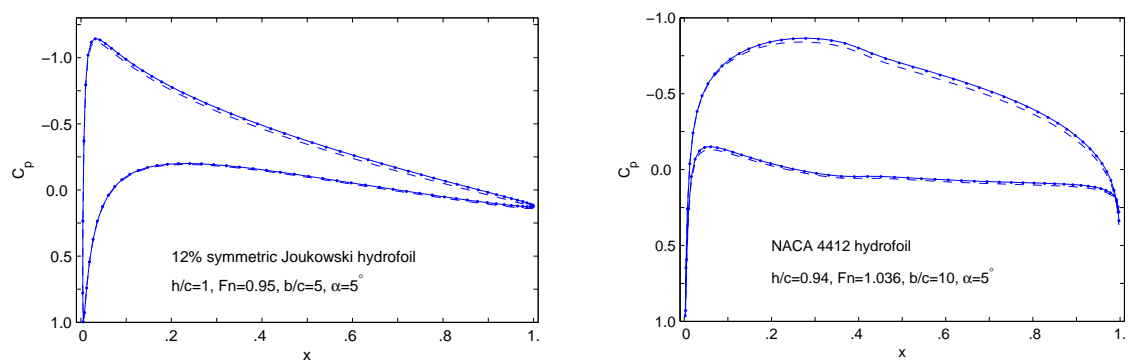

Figure 2. Pressure coefficient data calculated on the central plane $(y=0)$ pressure coefficients for $\mu=0.0001$ (solid line), $\mu=0.01$ (points line) and $\mu=0.1$ (dashed line).

The unknowns $\phi_{i, j}$ can be solved by Gaussian elimination algorithm. Hence we obtain the non-dimensional total velocity potential

$$
\Phi_{i, j}=\mathbf{q}_{i, j} \cdot(1,0,0)+\phi_{i, j}
$$

and the non-dimensional orthogonal tangential velocities

$$
v_{i, j}^{c}=\frac{\Phi_{i+1, j}-\Phi_{i, j}}{\left|\mathbf{p}_{i+1, j}-\mathbf{p}_{i, j}\right|}, \quad v_{i, j}^{s}=\frac{\Phi_{i, j+1}-\Phi_{i, j}}{\left|\mathbf{p}_{i, j+1}-\mathbf{p}_{i, j}\right|} .
$$

These tangential velocities determine the pressure coefficient on the hydrofoil surface panels

$$
C_{p}\left(\mathbf{q}_{i, j}\right)=\frac{\frac{1}{2} U^{2}-\frac{1}{2}\left|\nabla \hat{\Phi}\left(\mathbf{q}_{i, j}\right)\right|^{2}}{\frac{1}{2} U^{2}}=1-\left(v_{i, j}^{s}\right)^{2}-\left(v_{i, j}^{c}\right)^{2} .
$$

The lift and drag coefficients of the $j$ th cross section are defined as

$$
\begin{aligned}
C_{l} & =-\sum_{i=1}^{N_{c}} C_{p}\left(\mathbf{q}_{i, j}\right) \mathbf{n}_{i, j} \cdot(0,0,1)\left|\mathbf{p}_{i+1, j}-\mathbf{p}_{i, j}\right|, \\
C_{d} & =-\sum_{i=1}^{N_{c}} C_{p}\left(\mathbf{q}_{i, j}\right) \mathbf{n}_{i, j} \cdot(1,0,0)\left|\mathbf{p}_{i+1, j}-\mathbf{p}_{i, j}\right| .
\end{aligned}
$$

To facilitate comparisons of the numerical results with the experimental data $[22,23]$, the hydrofoil shapes are selected in the form of a NACA4412 and a $12 \%$ symmetric Joukowski contour operating at the angle attack $\alpha=5^{\circ}$ and various values of submergence-to-chord ratio $h / c$, span-to-chord ratio $b / c$ and Froude number $\mathrm{Fn}=U / \sqrt{g c}$. The submergence depth $h$ for the Joukowski shaped hydrofoil is measured as the vertical distance between the calm water surface $z=0$ and a trailing edge point $\mathbf{p}_{1, j}$ [23], whereas for the NACA4412 hydrofoil is measured as the vertical distance between the calm water surface and the middle chord point $[22]$.

In this study, the regular wave integral $K^{\mu}$ approximates the singular integral $\lim _{\mu \rightarrow 0+} K^{\mu}$ and hence only small $\mu$ values are considered. The present method is tested for $\mu$ in the interval $[0.0001,0.1]$ and it is found that the numerical pressure distribution on the body surface remains very stable as $\mu \rightarrow 0+$. The numerical data relate to the central plane $y=0$. This stability is demonstrated by the numerical pressure results illustrated in Figure 2, indicating that the differences in 

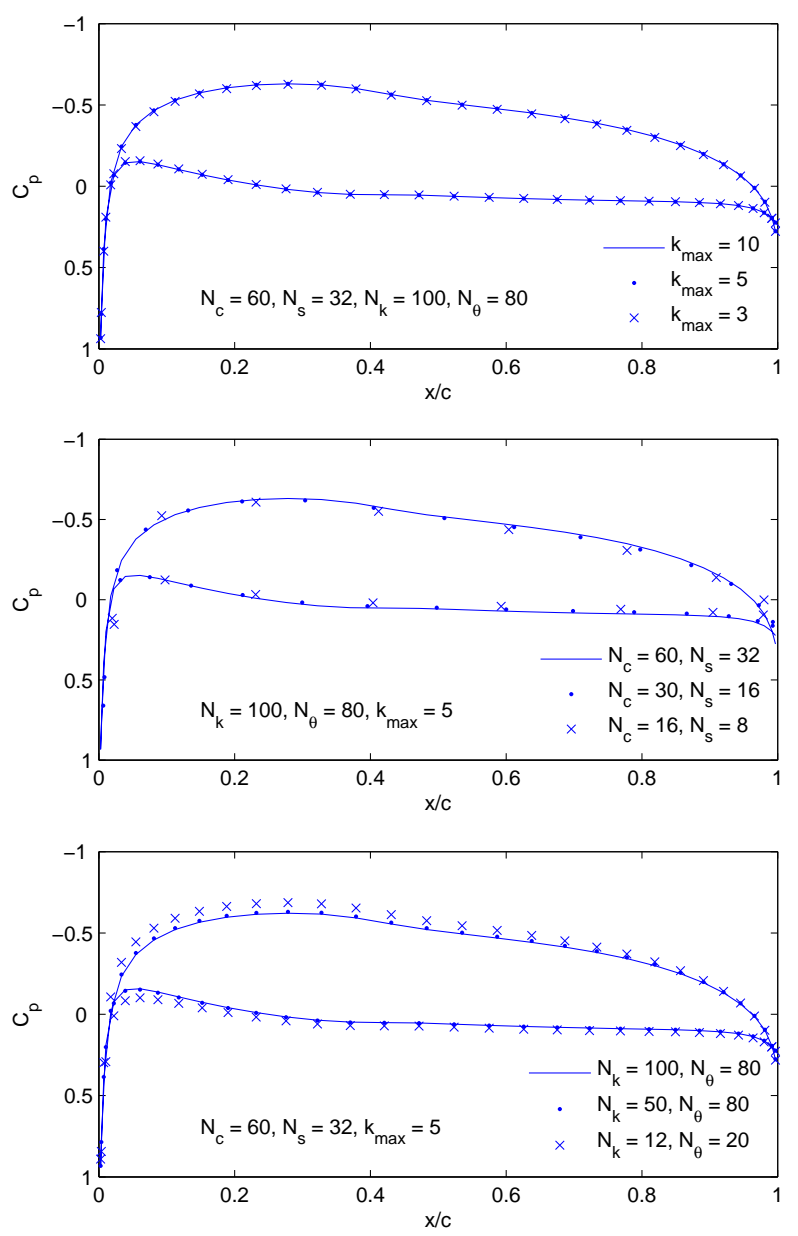

FIGURE 3. A comparison of pressure coefficient predictions calculated on the central cross section $(y=0)$ from the proposed three-dimensional mathematical model adopting different values of $k_{\max }, N_{k}, N_{\theta}, N_{c}$ and $N_{s}$ for a NACA4412 hydrofoil of $b / c=5$ operating at $F n=1.036, h / c=0.94, \alpha=5^{\circ}$ and $\mu=0.001$.

pressure coefficient between $\mu=0.1$ and $\mu=0.01$ are very small and indicating that the pressure coefficient is practically independent of $\mu$ in the region $\mu=0.01$ to $\mu=0.0001$. Therefore, in subsequent numerical simulations, we assume $\mu=0.001$. The numerical parameter values

$$
N_{c}=100, N_{s}=32, N_{k}=200, N_{\theta}=100, k_{\max }=10
$$

are generally selected in numerical simulations. Here $k_{\max }=k_{N_{k}+1}$ defines the finite wave number integral domain $\left[0, k_{\max }\right)$ approximating the infinite integral domain $[0, \infty)$ of $K^{\mu}$ in (3).

The values selected in (24) are large enough as the present method with respect to the increments of the values $k_{\max }, N_{k}, N_{\theta}, N_{c}$ and $N_{s}$ is very stable. This is demonstrated in Figure 3 for the selected results of a NACA4412 hydrofoil. 

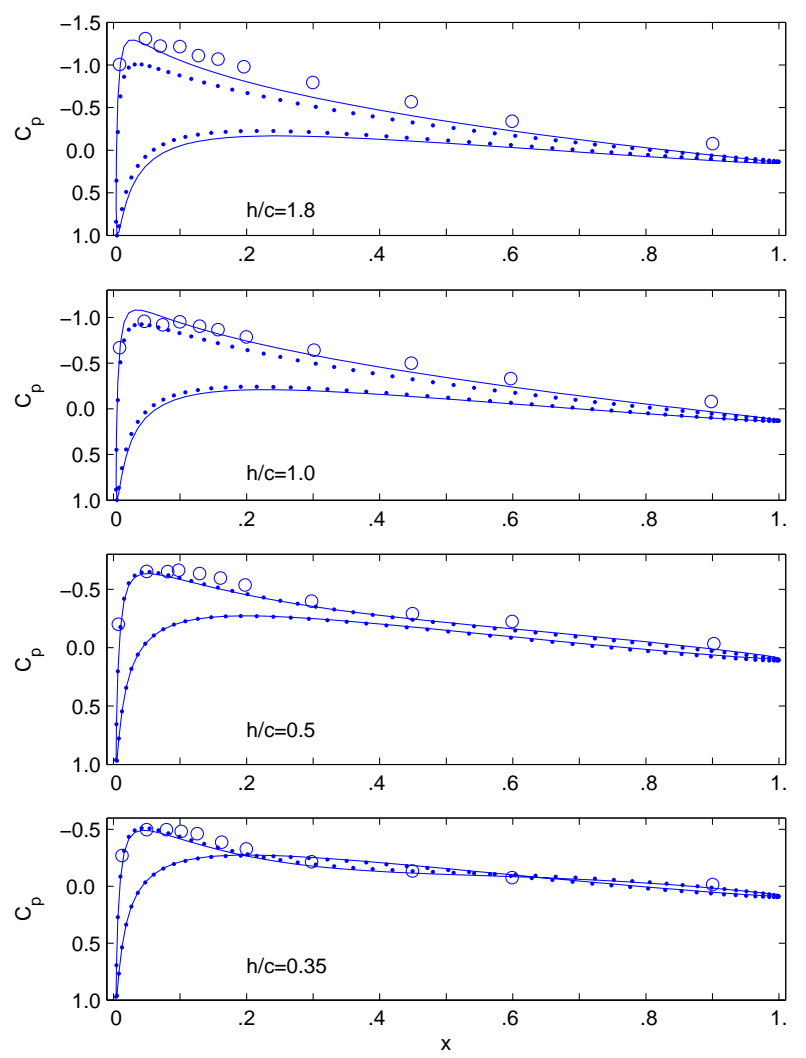

Figure 4. Pressure coefficient data with the central plane $(y=0)$ of a $12 \%$ symmetric Joukowski shaped hydrofoil $\left(\alpha=5^{\circ}, \mathrm{Fn}=\right.$ 0.95 ) compared to experimental data $[23]$ for $b / c=3.5$ (circle lines), calculations derived from the present method for $b / c=3.5$ and $\mu=0.001$ (solid line) and for $b / c=1.75$ and $\mu=0.001$ (point line).

A series pressure coefficient measurements [23] were recorded on the upper surface of a $12 \%$ symmetric Joukowski hydrofoil model $(b / c=3.5)$ held stationary in a water tunnel. Results derived from the present mathematical model are compared with the experimental data of [23] as depicted in Figure 4 for various submergence values and fixed Froude number. Figure 4 shows the dependence of the pressure distribution on the central plane $(y=0)$ to the span-to-chord ratio $b / c$ and the submergence-to-chord ratio $h / c$. It was further found that when the ratio $h / c \leq 0.35$ free-surface effects become more dominant and the pressure coefficient $C_{p}$ along the central plane $y=0$ is almost independent of the ratio $b / c \geq 1.75$ as demonstrated in Figure 4). When $h / c$ increases from $h / c=0.35$ to $h / c=1.8$, the free-surface influence reduces and the deviations of the pressure coefficient distributions increases along the central plane as observed in a comparison of findings between $b / c=1.75$ (point line) and $b / c=3.5$ (solid line) in Figure 4 for cases $h / c=1$ and $h / c=1.8)$. 

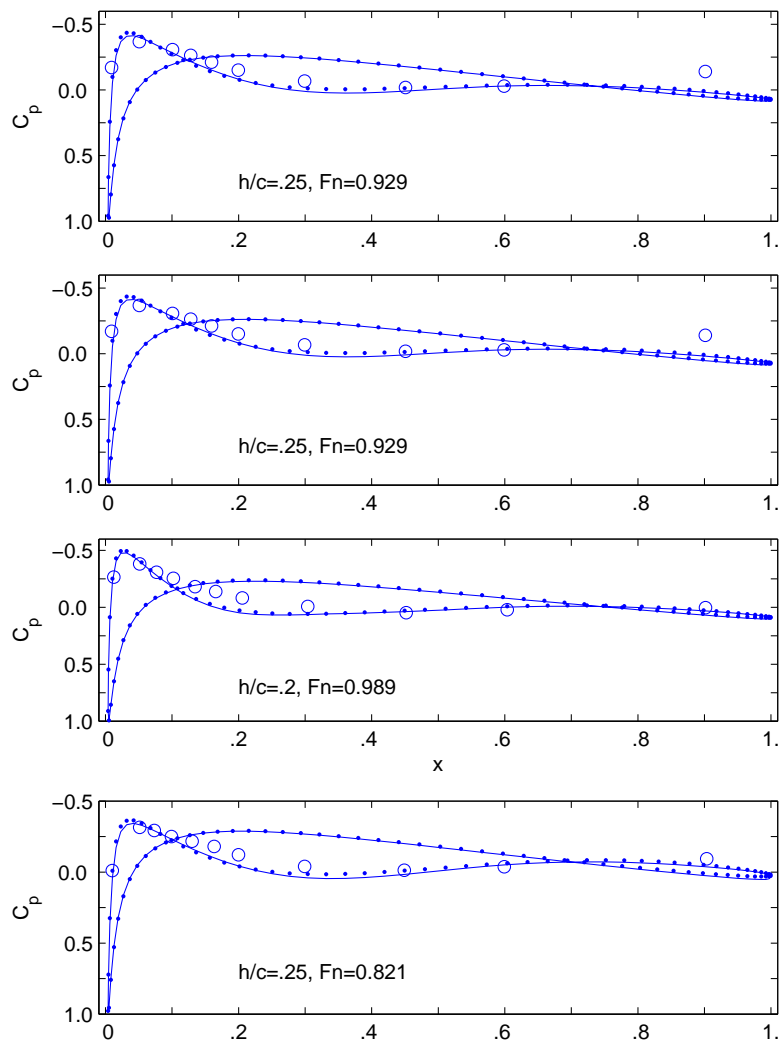

Figure 5. A comparison of pressure coefficient data on the central plane $(y=0)$ pressure coefficients of a $12 \%$ symmetric Joukowski shaped hydrofoil $\left(\alpha=5^{\circ}, b / c=3.5\right)$ between experimental data [23] assuming $b / c=3.5$ (circle line), predictions based on a twodimensional method [15] with $\mu=0.001$ (point line), and the described three-dimensional method for $\mu=0.001$ and $b / c=3.5$ (solid line).

It should be noted that the integrands of $K^{\mu}$ and $K$ in equations (3) and (4), respectively, contain implicitly the function $\mathrm{e}^{-k h / c}$, which decays exponentially for the submergence-to-chord ratio $h / c$. Thus its discretisation form $\mathrm{e}^{-k_{i+1} h / c}$ dominates the convergence of the expansions (18)-(19). Figure 5 illustrates a comparison of results obtained from the present method, experimental data and calculations derived from the corresponding two-dimensional method for small values of $h / c$ and various Fn values. It is seen that even when the hydrofoil is very near to the free surface, the results of the proposed method agree very well with the experimental data. The pressure coefficient data derived from a two-dimensional method [15] for a two-dimensional $12 \%$ symmetric hydrofoil corresponds to the predictions of the pressure coefficient on $y=0$ for a three-dimensional hydrofoil with $b / c=\infty$. Figure 5 indicates that central plane pressure distribution for $b / c=3.5$ is almost the same as that for $b / c=\infty$ when the submerged hydrofoils are very close to the free surface. 

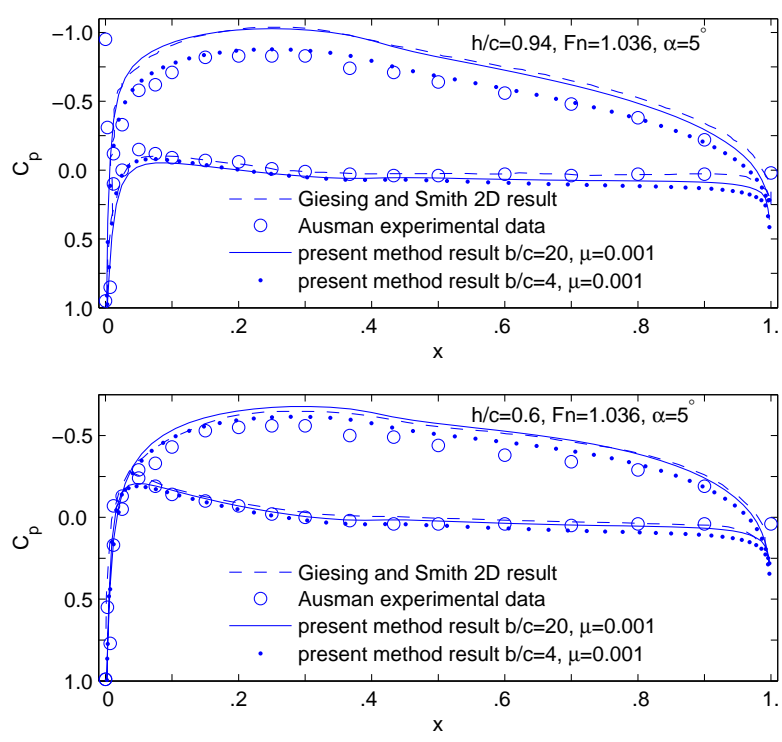

Figure 6. A comparison of pressure coefficient data from twodimensional based calculations, experimental data and predictions on the central cross section $y=0$ from the proposed threedimensional mathematical model adopting different $b / c$ values.

For the NACA4412 hydrofoil, the experimental data [22, Table II] relates to a model of $0.5 \mathrm{ft}$ chord mounted in a circulating water tunnel with uniform stream speed $U=4.15 \mathrm{ft} / s$. As assumed in [39], we take the submergence depths $h=0.47 \mathrm{ft}$ and $h=0.3 \mathrm{ft}$ giving a Froude number and submergence-to-chord ratios

$$
\mathrm{Fn}=\frac{U}{\sqrt{c g}}=1.036 \text { and } \frac{h}{c}=0.94,0.6
$$

Figure 6 illustrates a comparison of the results from the proposed method with measured data [22]. No span data was provided [22], and, therefore, two span values are assumed in the calculations obtained by the present method. The results demonstrate that the three-dimensional numerical model with the long span $(b / c=20)$ is able to describe favourably the calculated findings derived from a two-dimensional method [39, Figure 9]. However, the results suggest that the moderate span length $(b / c=4)$ may have been used in the water tunnel experiments [22]. The measured experimental data lie below the two-dimensional calculated values presented in [39, page 124]. It was confirmed that this difference is expected because the effect of the boundary-layer displacement reduces the circulation and thus the minimum pressure. However Figure 6 suggests that the major contributor to the difference of values is the ratio $b / c$ in the numerical and experimental scenarios rather than the effect of boundary-layer displacement. For the NACA4412 hydrofoil operating at $\alpha=5^{\circ}, h / c=0.94$ and $\mathrm{Fn}=1.036$, to produce good agreement between experimental data and two-dimensional numerical data, a large span-to-chord ratio value $(b / c \approx 20)$ hydrofoil model is required, which is not a radical proposition to use in 

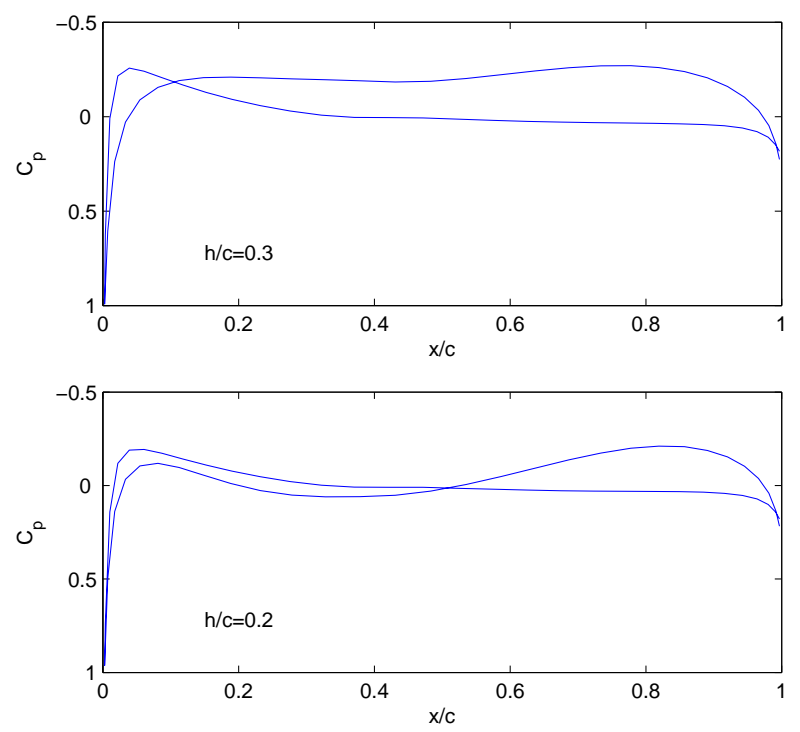

Figure 7. Pressure coefficient predictions calculated on the central cross section $(y=0)$ from the proposed three-dimensional mathematical model adopting different values of $h / c$ for a NACA4412 hydrofoil of $b / c=5$ operating at $F n=0.9, \alpha=5^{\circ}$ and $\mu=0.001$.

experiments and in a towing tank or water tunnel. However, if the submergenceto-chord ratio $h / c=0.6$, the span-to-chord $b / c$ value for an experimental model can be reduced significantly due to the increment of free-surface effects.

The numerical simulations for a NACA4412 hydrofoil for the shallow submergence case are displayed in Figure 7 . The distance between the calm water surface and the hydrofoil is 0.085 when $h / c=0.2$ and is 0.1854 when $h / c=0.3$. With the decrease of the $h / c$ value, the upper surface pressure coefficient line turns to intersect with the lower surface one at a point away from trailing and leading edges.

Lift coefficient $C_{l}$ and drag coefficient $C_{d}$ data are determined from the threedimensional mathematical model for a NACA4412 hydrofoil with the submergence $h / c=1$ and Froude numbers $0.4 \leq \mathrm{Fn} \leq 1.8$. These are compared to numerical data [31] relating to two-dimensional flows. The calculations of the coefficients $C_{l}$ and $C_{d}$ from the three-dimensional model produce convergent solutions with respect to the increment of span-to-chord ratio $b / c$. Figure 8 displays results for $C_{l}$ and $C_{d}$ on the central plane $y=0$ for a variety of values of $b / c$. These show that the present method produces predictions favourably compared to corresponding two-dimensional numerical data [31] when $b / c \geq 15$.

\section{NumERiCAL SIMULATION FOR FREE-SURFACE WAVES}

The free-surface wave expression to be discussed herein is related to far field free-surface wave formulations (see, for example, $[18,19,21,20]$ ) of Kelvin waves. It is necessary to refresh the previous formulations to understand their differences. 

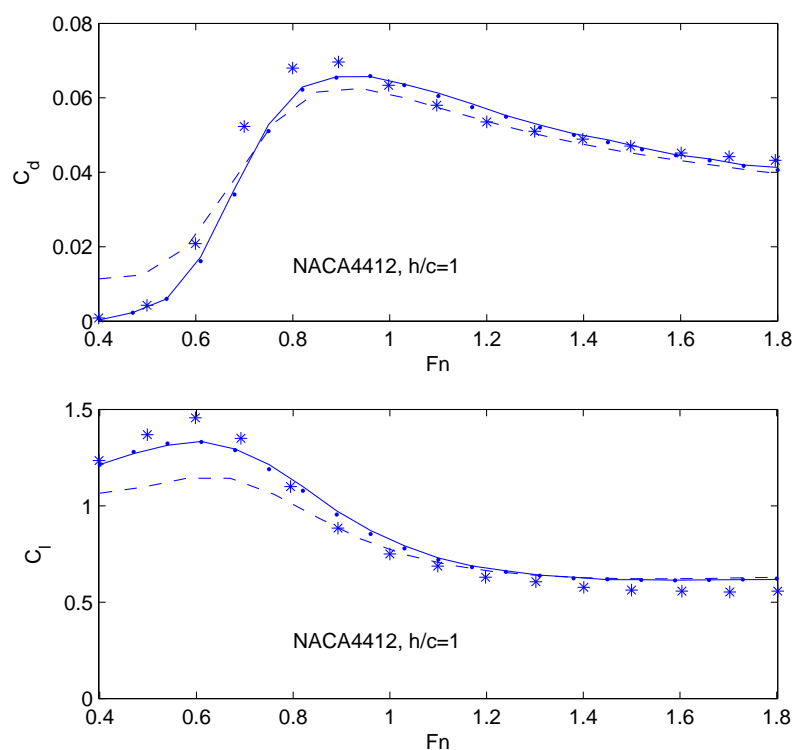

Figure 8. Calculated lift and drag coefficient data associated with a two-dimensional hydrofoil comparing a two-dimensional method [15] (asterisk line) and the presented method $(\mu=0.001)$ evaluated on the central plane $(y=0)$ of a three-dimensional hydrofoil with parameters $b / c=8$ (dash line), $b / c=15$ (point line) and $b / c=20$ (solid line).

From the Green function expression (2), the free-surface wave generated by the advancing source point $(\xi, \eta, \zeta)=(0,0,0)$ is combined by the far field wave defined by the single integral of (2) and a near field wave defined by the double integral of (2). Therefore a far field wave is integrated by one-dimensional waves in the directions $-\frac{\pi}{2}<\theta<\frac{\pi}{2}$ (see, for example, [21]) as given by the single integral of (2) expressed as

$$
\begin{gathered}
4 \nu \int_{0}^{\frac{\pi}{2}} \mathrm{e}^{\nu z \sec ^{2} \theta} \sin [\nu x \sec \theta] \cos \left[\nu y \sin \theta \sec ^{2} \theta\right] \sec ^{2} \theta d \theta \\
=-4 \nu \operatorname{Re} \int_{-\frac{\pi}{2}}^{\frac{\pi}{2}} \mathrm{ie}^{\nu \sec ^{2} \theta[z+\mathrm{i} x \cos \theta+\mathrm{i} y \sin \theta]} \sec ^{2} \theta d \theta
\end{gathered}
$$

This consideration dates back to Havelock $[18,19]$ on the expression of the far field free-surface wave

$$
\begin{aligned}
\chi= & \int_{-\frac{\pi}{2}}^{\frac{\pi}{2}} a_{1}(\theta) \sin \left[\nu \sec ^{2} \theta(x \cos \theta+y \sin \theta)\right] d \theta \\
& +\int_{-\frac{\pi}{2}}^{\frac{\pi}{2}} a_{2}(\theta) \cos \left[\nu \sec ^{2} \theta(x \cos \theta+y \sin \theta)\right] d \theta
\end{aligned}
$$

integrated by the elementary waves

$$
a_{1}(\theta) \sin \left[\nu \sec ^{2} \theta(x \cos \theta+y \sin \theta)\right]
$$


and

$$
a_{2}(\theta) \cos \left[\nu \sec ^{2} \theta(x \cos \theta+y \sin \theta)\right]
$$

for wave amplitudes $a_{1}$ and $a_{2}$.

In the present study, we consider the complete formulation of the free-surface wave, which is the summation of the far field free-surface wave part and the near field free-surface wave part. The former represents the integral of the elementary waves for $-\frac{\pi}{2}<\theta \leq \frac{\pi}{2}$ at the single wave number value $k=\nu$ and is determined by the single integral of (2). The latter, defined by the double integral of (2), represents for the integral of the elementary waves for $-\frac{\pi}{2}<\theta \leq \frac{\pi}{2}$ and $k>0$ but $k \neq \nu$. Therefore, for the consideration of the whole free-surface waves, it is suitable to use the double integral (3) instead of (2) to get a straightforward discretisation of free-surface waves due to the absence of singularity in (3).

It follows from the Bernoulli equation and (8) that the linearized surface wave elevation is expressible in the form

$$
\chi=\frac{1}{g}\left(\frac{1}{2}\left|\partial_{x^{\prime}} \hat{\Phi}\right|^{2}-\frac{U^{2}}{2}\right)=-c \mathrm{Fn}^{2} \partial_{x} \phi \text { for } z=0 .
$$

For a field point $\mathbf{q}=(x, y, 0)$ on the calm water surface, the Rankine source and its image cancel one another. It follows from (12), (14) and (17) that

$$
\begin{aligned}
\partial_{x} \phi(\mathbf{q})= & -\sum_{i=1}^{N_{c}+1} \sum_{j=1}^{N_{s}} \frac{\phi_{i, j}}{4 \pi} \int_{\text {panel }_{i, j}} \mathbf{n}_{i, j} \cdot \nabla \partial_{x} K^{\mu}(\mathbf{q}, \mathbf{p}) d s_{\mathbf{p}} \\
& -\sum_{i=1}^{N_{c}} \sum_{j=1}^{N_{s}} \frac{n_{i, j, 1}}{4 \pi} \int_{\text {panel }_{i, j}} \partial_{x} K^{\mu}(\mathbf{q}, \mathbf{p}) d s_{\mathbf{p}} \\
= & -\sum_{i=1}^{N_{c}} \sum_{j=1}^{N_{s}} \frac{\mid \text { panel }_{i, j} \mid}{4 \pi} \\
& \cdot\left[\phi_{i, j} \mathbf{n}_{i, j} \cdot \nabla \partial_{x} K^{\mu}\left(\mathbf{q}, \mathbf{p}_{i, j}\right)+n_{i, j, 1} \partial_{x} K^{\mu}\left(\mathbf{q}, \mathbf{p}_{i, j}\right)\right] \\
& -\sum_{j=1}^{N_{s}} \frac{\phi_{N_{c}+1, j}}{4 \pi} \int_{\mathbf{p}_{N_{c}+1, j}}^{\mathbf{p}_{N_{c}+2, j}} \int_{\mathbf{p}_{N_{c}+1, j}}^{\mathbf{p}_{N_{c}+1, j+1}} \\
& \mathbf{n}_{N_{c}+1, j} \cdot \nabla \partial_{x} K^{\mu}(\mathbf{q}, \mathbf{p}) d s,
\end{aligned}
$$

where $\mid$ panel $_{i, j} \mid$ denotes the area of panel $_{i, j}$.

Combining the previous equation with the expansion [16], we find that

$$
\begin{aligned}
& \frac{\partial K^{\mu}(\mathbf{q}, \mathbf{p})}{\partial x} \\
& =4 \pi \nu \operatorname{Re} \sum_{l=1}^{N_{k}} \sum_{m=1}^{N_{\theta}} \hat{c}_{l, m} \mathrm{e}^{k_{l} \zeta+\mathrm{i} k_{l}\left[(x-\xi) \cos \theta_{m}+(y-\eta) \sin \theta_{m}\right] .}
\end{aligned}
$$

Here the the expansion coefficients are expressed as

$$
\begin{aligned}
\hat{c}_{l, m}= & \frac{1}{2 \pi^{2}} \mathrm{i} k_{l} \ln \frac{k_{l+1} \cos ^{2} \theta_{m}-\nu-\mathrm{i} \mu \cos \theta_{m}}{k_{l} \cos ^{2} \theta_{m}-\nu-\mathrm{i} \mu \cos \theta_{m}} \\
& \cdot \ln \frac{\left(1+\tan \frac{\theta_{m+1}}{2}\right)\left(1-\tan \frac{\theta_{m}}{2}\right)}{\left(1+\tan \frac{\theta_{m}}{2}\right)\left(1-\tan \frac{\theta_{m+1}}{2}\right)}
\end{aligned}
$$


such that

$$
\begin{aligned}
\partial_{x} \phi(\mathbf{q}) & \\
= & -\operatorname{Re} \sum_{i=1}^{N_{c}} \sum_{j=1}^{N_{s}} \sum_{l=1}^{N_{k}} \sum_{m=1}^{N_{\theta}} \frac{\left|\mathrm{panel}_{i, j}\right|}{\mathrm{Fn}^{2}} \hat{c}_{l, m} \\
& \cdot\left[k_{l} \phi_{i, j} \mathbf{n}_{i, j} \cdot\left(-\mathrm{i} \cos \theta_{m}, 0,1\right)+n_{i, j, 1}\right] \\
& \cdot \mathrm{e}^{k_{l} \zeta_{i, j}+\mathrm{i} k_{l}\left[\left(x-\xi_{i, j}\right) \cos \theta_{m}+\left(y-\eta_{i, j}\right) \sin \theta_{m}\right]} \\
& -\operatorname{Re} \sum_{j=1}^{N_{s}} \sum_{l=1}^{N_{k}} \sum_{m=1}^{N_{\theta}} \frac{\left(\eta_{N_{c}+1, j+1}-\eta_{N_{c}+1, j}\right)}{\mathrm{Fn}^{2}} \phi_{N_{c}+1, j} \hat{c}_{l, m} \\
& \cdot \frac{1-\mathrm{i} \cos \theta_{m}}{1+\mathrm{i} \cos \theta_{m}} \\
& \cdot \mathrm{e}^{k_{l} \zeta_{N_{c}+1, j}+\mathrm{i} k_{l}\left[\left(x-\xi_{N_{c}+1, j}\right) \cos \theta_{m}+\left(y-\eta_{N_{c}+1, j}\right) \sin \theta_{m}\right]} .
\end{aligned}
$$

If we simply rewrite the coefficients in the form

$$
\begin{aligned}
& c_{i, j, l, m}= \\
& \quad\left|\operatorname{panel}_{i, j}\right| \hat{c}_{l, m}\left[k_{l} \phi_{i, j} \mathbf{n}_{i, j} \cdot\left(-\mathrm{i} \cos \theta_{m}, 0,1\right)+n_{i, j, 1}\right] \mathrm{e}^{k_{l} \zeta_{i, j}}
\end{aligned}
$$

for $i \leq N_{c}$,

$$
\begin{aligned}
& c_{i, j, l, m}= \\
& \quad\left(\eta_{N_{c}+1, j+1}-\eta_{N_{c}+1, j}\right) \phi_{N_{c}+1, j} \hat{c}_{l, m} \frac{1-\mathrm{i} \cos \theta_{m}}{1+\mathrm{i} \cos \theta_{m}} \mathrm{e}^{k_{l} \zeta_{N_{c}+1, j}}
\end{aligned}
$$

for $i=N_{c}+1$, the previous formulation reduces to

$$
\begin{aligned}
\partial_{x} \phi(\mathbf{q})= & -\frac{1}{\mathrm{Fn}^{2}} \operatorname{Re} \sum_{i=1}^{N_{c}+1} \sum_{j=1}^{N_{s}} \sum_{l=1}^{N_{k}} \sum_{m=1}^{N_{\theta}} c_{i, j, l, m} \\
& \cdot \mathrm{e}^{\mathrm{i} k_{l}\left[\left(x-\xi_{i, j}\right) \cos \theta_{m}+\left(y-\eta_{i, j}\right) \sin \theta_{m}\right]}
\end{aligned}
$$

It follows from (25) that the Kelvin wave is expressible as an expansion of plane regular waves in the form

$$
\begin{aligned}
\underline{\chi}= & \operatorname{Re} \sum_{i=1}^{N_{c}+1} \sum_{j=1}^{N_{s}} \sum_{l=1}^{N_{k}} \sum_{m=1}^{N_{\theta}} c_{i, j, l, m} \\
& \cdot \mathrm{e}^{\mathrm{i} k_{l}\left[\left(x-\xi_{i, j}\right) \cos \theta_{m}+\left(y-\eta_{i, j}\right) \sin \theta_{m}\right]}
\end{aligned}
$$

Figure 9 displays numerical results of the two-dimensional surface wave generated by (28) on the central plane $y=0$ for a submerged NACA4412 hydrofoil of span-tochord ratio values $b / c=4,8,20$ traveling at Froude number, Fn $=0.9, \alpha=5^{\circ}$ and $h / c=1$. It also illustrates the surface wave on the plane $z=0$ calculated by the three-dimensional theory described herein. In the three-dimensional theory, taking $b / c=20$, the surface wave generated on the central plane is in a good agreement with the two-dimensional numerical surface wave given by [31]. However, when the ratio reduces to $b / c=8$ and 4 , the central plane $(y=0)$ waves are significantly different to the two-dimensional flow wave due to wave radiation damping.

From equation (28) and its derivation, it follows that the wave elevation can be expanded as stationary propagating regular waves (see Figure 10) of wave numbers 

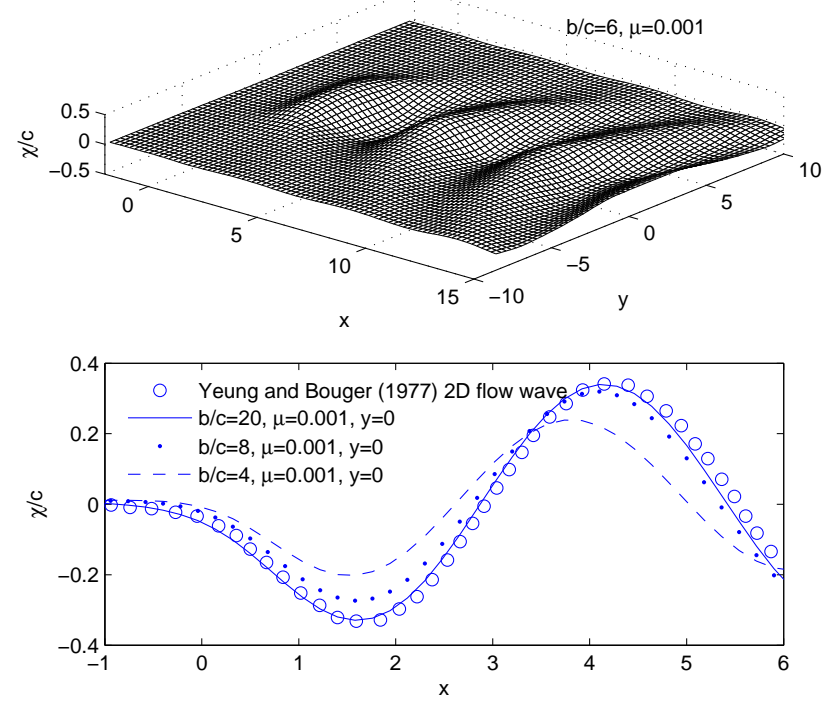

FiguRE 9. Generated three-dimensional surface waves, and twodimensional surface waves on the central plane $y=0$ compared to a calculated two-dimensional surface wave [31] for a NACA4412 hydrofoils operating at $\mathrm{Fn}=0.9, h / c=1, \alpha=5^{\circ}$.
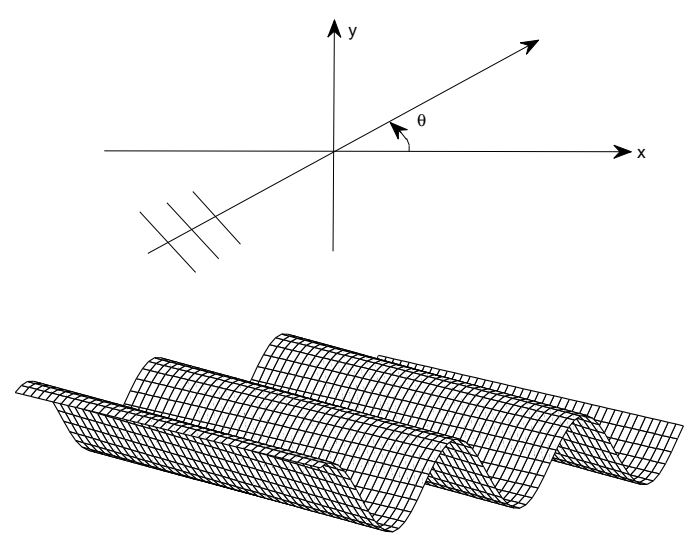

Figure 10. Profile of a single stationary propagating regular wave for $k>0$ and $\theta \in\left(-\frac{\pi}{2}, \frac{\pi}{2}\right)$.

$k>0$ and incident wave angles $-\frac{\pi}{2}<\theta<\frac{\pi}{2}$ centred at mesh grid points of hydrofoil surface. Moreover, the derivation of the wave expansion (28) shows that the surface wave $\chi$ is sum of surface waves produced by the sources located on hydrofoil panels. 
To facilitate the understanding of the wave expansion, we employ (26) to derive the single source wave $\chi_{s}$ expressible as

$$
\begin{aligned}
\frac{\chi_{s}}{h} & =-\mathrm{Fn}^{2} \frac{\mathcal{M}}{U h^{2} 4 \pi} \frac{\partial K^{\mu}}{\partial x} \\
& =\frac{\mathcal{M}}{U h^{2}} \operatorname{Re} \sum_{l=1 m=1}^{N_{k}} \sum_{N_{\theta}}^{N_{l}} \hat{c}_{l, m} \mathrm{e}^{k_{l} \zeta+\mathrm{i} k_{l}\left[(x-\xi) \cos \theta_{m}+(y-\eta) \sin \theta_{m}\right]}
\end{aligned}
$$

for strength $\mathcal{M}$, submergence depth $h$ and speed $U$. Here the typical length $c$ of the flow is set to be $h$ and so the source point $(\xi, \eta, \zeta)$ can be set as $(0,0,-1)$. Following [40], we choose

$$
\frac{\mathcal{M}}{U h^{2}}=2.6 \text { and } \mathrm{Fn}=0.7
$$

The wave expansion (29) has been validated [16] through comparison with the numerical data of [40]. Let us now consider partial summations of the regular waves with respect to $k=k_{l} \in(0, \infty)$ and $\theta=\theta_{m} \in\left(-\frac{\pi}{2}, \frac{\pi}{2}\right)$ to analyse the structure of source wave shown in Figure 11.

The wave in Figure 11 (a) is determined exactly by (29) through the complete summation of regular waves $(y>0)$ for $k_{l}>0$ and $-\frac{\pi}{2}<\theta_{m}<\frac{\pi}{2}$. The wave in Figure $11(\mathrm{~b})$ is the summation of regular waves for all wave numbers $k=k_{l}>0$ and the partial angle values $\theta=\theta_{m} \in\left(-\frac{\pi}{2},-\frac{\pi}{2}+\frac{3 \pi}{20}\right)$. This implies that these regular waves mainly contribute to the formation of divergent waves. Figure 11 (c) shows that the regular waves with respect to $\theta=\theta_{m}$ around 0 gives rise to transverse waves of the Kelvin wave in Figure 11 (a).

Moreover, to see the role of the wave number $k$, the component parts of the summations of regular waves for all $\theta=\theta_{m} \in\left(-\frac{\pi}{2}, \frac{\pi}{2}\right)$ and partial $k=k_{l}>0$ based on the formula (29) are illustrated in Figure 11 (d)-(e). These figures imply that the regular waves with small wave numbers or large wave lengthes have little contribution to the formation of Kelvin wave in Figure 11 (a). When the partial summation is made for the regular waves with $k \leq 2.8$, the resultant wave is very close to Figure 11 (a). That is, the regular waves with large wave numbers can be ignored without causing a significant reduction of the accuracy of the result. It was found that main contributors to the Kelvin wave are by regular waves with $k=k_{l}$ around the wave number $\nu=2.04$.

In similar fashion to the source waves discussed in Figure 11, the partial summations of regular waves with respect to $\theta=\theta_{m}$ and $k=k_{l}$ based on the plane regular wave expansion formula (28) are displayed in Figure 12. The construction of the wave (28) is much more complex than that of the single source wave (29). However, some properties are shared by these two kind of waves. For example, it is implied in Figure 11 that the regular waves with small or large wave numbers $k=k_{l}$ are not important in the formation of the Kelvin wave illustrated in Figure 9 and the regular waves with $\theta=\theta_{m}$ close to 0 are main contributors to the formation of the divergent waves.

\section{Discussion}

Steady free-surface potential flow around a lifting body is traditional problem in hydrodynamics and has been well studied in both linear (see, for example,[8, 39]) 

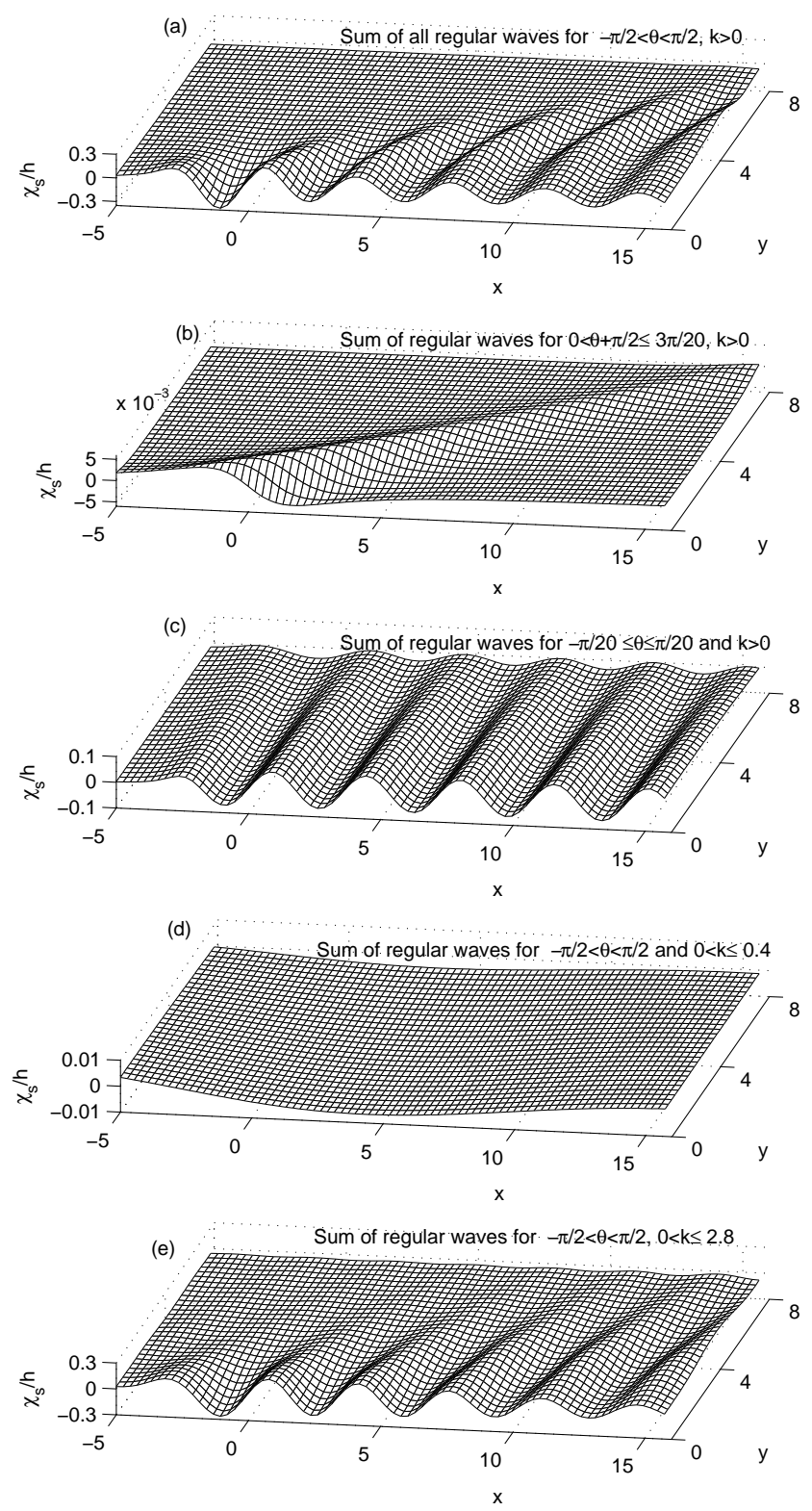

Figure 11. Partial summations of regular waves $(y>0)$ of a single source operating at $\mathrm{Fn}=0.7$ and $\mathcal{M} /\left(U h^{2}\right)=2.6$ based on the summation formula (29) for $\theta=\theta_{m}$ and $k=k_{l}$.

and nonlinear (see, for example, $[40,42]$ ) aspects. Even for the linear threedimensional free-surface problem, the free-surface Green function approach is still widely accepted as a difficult problem due to singular wave integral $K=\lim _{\mu \rightarrow 0+} K^{\mu}$ presented in (3). 

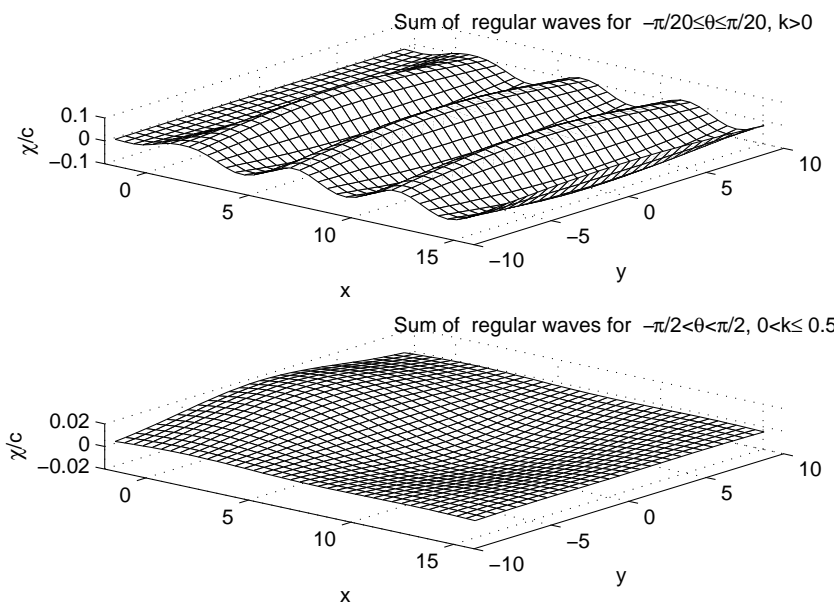

Sum of regular waves for $-\pi / 2<\theta<\pi / 2,0<k \leq 5$

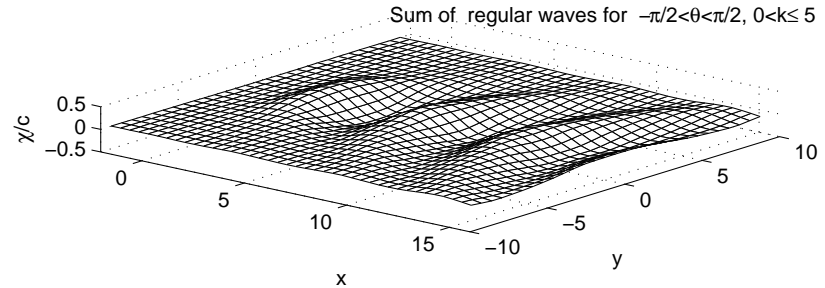

FigURE 12. Partial summations of regular waves of a NACA4412 hydrofoil operating at $\mathrm{Fn}=0.9$ and $h / c=1$ based on the summation formula (28) for $\theta=\theta_{m}$ and $k=k_{l}$.

It is demonstrated in the present study for a lifting body problem that the singularity can be ignored by using $K^{\mu}$ for $\mu=0.001$ or $\mu=0.0001$ in place of $K$, which was evaluated after taking the limit $\mu \rightarrow 0+$ in earlier investigations. Numerically, there is no difference between $K^{\mu=0.0001}$ and $\lim _{\mu \rightarrow 0+} K^{\mu}$ with respect to engineering problems. However, the former is a regular integral, while the latter is singular integral. This approximation scheme developed from $[14,15,16,17]$ helps introduce whole space harmonic function expansions of the half space harmonic function $K^{\mu=0.0001} \approx K$ and then present a simple and straightforward free-surface Green function based panel method for the lifting body problem. The extension of this method to the translating and pulsating free-surface Green function was recently developed in [43].

With the use of the harmonic function expansions, free water surface generated by the movement of the hydrofoil in the potential flow is expressed as an expansion of plane regular waves for selected $\theta$ directions and wave number $k$ values. This understanding is different to the Havelock elementary wave theory [18, 19, 20] showing the far field wave part at the single wave number value $k=\nu$.

Present method results are in very good agreement with experimental data of $[22,23]$ and the numerical results of $[15,39]$.

Acknowledgment. This research was partially supported by NSF (grant No. 11571240) of China. 


\section{REFERENCES}

[1] Bal S, Kinnas SA, Lee H (2001) Numerical analysis of 2-D and 3-D cavitating hydrofoils under a free surface. J Ship Res 45: 34-49

[2] Havelock TH (1928) Wave resistance. Proc R Soc Lond A 118: 24-33

[3] Wehausen JV, Laitone EV (1960) Surface waves. In: Flugge S, Truesdell C(Eds), Fluid Dynamics III in Handbuch der Physik 9, Springer, Berlin, pp. 446-778

[4] Baar JJM, Price WG (1988) Development in the calculation of wavemaking resistance of ships. Proc R Soc Lond A 416: 115-147

[5] Bessho M (1964) On the fundamental function in the theory of the wave-making resistance of ships. Mem Defense Academy of Japan 6:99-119

[6] Newman JN (1987) Evaluation of the wave-resistance Green function: Part 1 - The double integral. J Ship Res 31:79-90

[7] Hess JL, Smith AMO (1964) Calculation of nonlifting potential flow about arbitrary threedimensional bodies. J Ship Res 8: 22-44

[8] Hess JL, Smith AMO (1966) Calculation of potential flow about arbitrary bodies. Prog Aeronautical Sci 8: 1-138

[9] Noblesse F (1981) Alternative integral representations for the Green function of the theory of ship wave resistance. J Eng Math 15: 241-265

[10] Dawson CW (1977) A practical computer method for solving ship wave problems. In Proceedings of 2nd International Conference on Numerical Ship Hydrodynamics. University of California, Berkeley, pp. 30-38

[11] Havelock TH (1932) The theory of wave resistance. Proc R Soc Lond A 138: 339-348

[12] Lighthill J (1967) On waves generated in dispersive systems by traveling forcing effects, with applications to the dynamics of rotating fluids. J Fluid Mech 27: 725-752

[13] Noblesse F, Yang C (2007) Elementary water waves. J Eng Math 59:277-299

[14] Chen ZM (2012) A vortex based panel method for potential flow simulation around a hydrofoil. J fluids Strut 28: 378-391

[15] Chen ZM (2013) A dissipative free-surface approach for an underwater body in a uniform stream. J Marine Sci Tech 18: 182-191

[16] Chen ZM (2013) Harmonic function expansion for translating Green functions and dissipative free-surface waves. Wave Motion 50: 282-294

[17] Chen ZM (2014) Regular wave integral approach to the prediction of hydrodynamic performance of submerged spheroid, Wave Motion 51: 193-205.

[18] Havelock TH (1934) Wave patterns and wave resistance. Trans Inst Naval Arch 76: 430-446

[19] Havelock TH (1934) The calculation of wave resistance. Proc R Soc A 144: 514-521

[20] Inui T (1962) Wave-making resistance of ships. Presented at the Annual Meeting of The Society of Naval Architects and Marine Engineers, New York, November 15-16, 1962

[21] Tuck EO, Scullen DC, Lazauskas L (2001) Ship-wave patterns in the spirit of Michell. In: King AC, Shikhmurzaev Y (eds) IUTAM symposium on Free surface flows. Birmingham, UK, 2000, pp. 311-318

[22] Ausman JS (1954) Pressure limitation on the upper surface of a hydrofoil. PhD thesis, University of California, Berkeley.

[23] Parkin BR, Perry B, Wu TY (1956) Pressure distribution on a hydrofoil running near the water surface. J Appl Phys 27: 232-240

[24] Xie N, Vassalos D (2007) Performance analysis of 3D hydrofoil under free surface. Ocean Engineering 34: 1257-1264

[25] Tarafder MS, Saha GK, Mehedi ST (2010) Analysis of potential flow around 3-dimensional hydrofoils by combined source and dipole based panel method. J Marine Sci Tech 18: 376-384

[26] Liang H, Sun L, Zong L, Zhou L, Zou L (2013) Analytical modelling for a three-dimensional hydrofoil with winglets operating beneath a free surface. Appl Math Modelling 37: 2679-2701

[27] Carabineanu A (2014) The study of the potential flow past a submerged hydrofoil by the complex boundary element method. Engineering Analysis with Boundary Elements 39: 2335

[28] Huang F, Yang C, Noblesse F (2013) The Neumann-Michell theory of ship waves. J Eng Math $79: 5171$ 
[29] Noblesse F, Huang F, Yang C (2013) Numerical implementation and validation of the Neumann-Michell theory of ship waves. Eur J Mech B/Fluids 42: 47-68

[30] Wu H, Zhang C, Ma C, Huang F, Yang C, Noblesse F (2016) Errors due to a practical Green function for steady ship waves. Eur J Mech B/Fluids 55: 162-169

[31] Yeung RW, Bouger YC (1977) Hybrid integral-equation method for the steady ship-problem. In: Second International Conference on Numerical Ship Hydrodynamics, Berkeley, pp. 160175

[32] Yeung RW, Bouger YC (1979) A hybrid integral-equation method for steady two-dimensional ship waves. Int J Num Method Eng 14: 317-336

[33] Nakos DE (1990) Ship wave patterns and motions by a three dimensional Rankine panel method. PhD thesis, Massachusetts Institute of Technology, Cambridge

[34] Nakos DE, Sclavounos PD (1990) On steady and unsteady ship wave patterns. J Fluid Mech 215: $263-288$

[35] Beck RF (1994) Time-domain computations for floating bodies. Appl Ocean Res 16:267-282

[36] Longuet-Higgins M, Cokelet E (1976) The deformation of steep surface waves on water. I. a numerical method of computation. Proc R Soc Lond A 350: 1-26

[37] Longuet-Higgins M, Cokelet E (1978) The deformation of steep surface waves on water. II. growth of normal-mode instabilities. Proc R Soc Lond A 364: 1-28

[38] Newman JN (1986) Distributions of sources and normal dipoles over a quadrilateral panel. J Eng Math 20:113-126

[39] Giesing JP, Smith AMO (1967) Potential flow about two-dimensional hydrofoil. J Fluid Mech 28: $113-129$

[40] Forbes LK (1989) An algorithm for 3-dimensional free-surface problems in hydrodynamics. J Comput Phys 82: 330-347

[41] Katz J, Plotkin A (1991) Low-Speed Aerodynamics. McGraw-HIll, Inc, New York

[42] Raven HC (1996) A solution method for the nonlinear ship wave resistance problem. PhD thesis, Delft University of Technology, Delft

[43] Chen ZM (2014) Straightforward approximation of the translating and pulsating free surface green function, Discrete Contin. Dynam. Syst. B 19: 2767-2783

School of Mathematics and Statistics, Shenzhen University, Shenzhen 518052, China, Ship Science, University of Southampton, Burgess Road, Southampton So16 7QF, UK E-mail address, corresponding address: zhimin@soton.ac.uk

School of Mathematics and Statistics, Shenzhen University, Shenzhen 518052, China

Ship Science, University of Southampton, Burgess Road, Southampton SO16 7QF, UK, WUT-Uos High Performance Ship Technology Joint Center, Wuhan 430063, China 